Die Konferenz sowie die Festschrift für Prof. I.I Černyševa, die zu diesem Anlass erschienen ist, spiegeln nicht nur die aktuellen Fragen der Lexikologie und Phraseologieforschung wider. Sie leisten auch einen Beitrag zur Ehrung einer hervorragenden Wissenschaftlerin und zeigen, dass ihre Werke einen nachhaltigen wissenschaftlichen Impuls darstellen.

Für Organisation, Gestaltung und Durchführung der Konferenz soll an dieser Stelle den Veranstaltern ein großer Dank ausgesprochen werden - das war eine sehr gelungene Tagung mit wunderbarer Atmosphäre.

Agnieszka Błażek

\title{
Bericht über die internationale Konferenz Fremdsprachenunterricht - omnimedial?, Poznań 25.-26. 03. 2011
}

Im Institut für germanische Philologie der Adam-Mickiewicz-Universität in Poznań fand am 25.-26. März 2011 eine internationale Konferenz statt, deren Titel Fremdsprachenunterricht - omnimedial? lautete. Für ihre Organisation waren die Mitarbeiter des Lehrstuhls für Methodik und Didaktik DaF zuständig.

Folgende thematische Schwerpunkte bildeten den Rahmen der Tagung:

- Fremdsprachenlernen in medialen Umgebungen - aktivierend, motivierend, bereichernd oder ...?

- Lernplattformen, E-Mail-Klassenpartnerschaften und -Tandems, Podcasts, Blogs, Filme, Internetrecherchen, elektronische Nachschlagewerke, interaktive Lernangebote auf CD-ROM und online... - bloß Medienterror oder sinnvolle Bereicherung des Unterrichtsalltags?

- Förderung der interkulturellen kommunikativen Kompetenz durch den Einsatz von authentischen bzw. für Unterrichtszwecke präparierten Medientexten,

- Förderung der Lernerautonomie durch den Umgang mit Medien,

- Medienkompetenz als Voraussetzung erfolgreicher Arbeit mit Medien im Unterricht - Anforderungen an Lehrkräfte und Lernende, Möglichkeiten der Förderung und Messung.

$\mathrm{Zu}$ Beginn der Konferenz wurden alle Teilnehmer von Prof. Dr. habil. Kazimiera Myczko, der Leiterin des Lehrstuhls für Methodik und Didaktik DaF, und Prof. Dr. habil. Czesław Karolak, dem Direktor des Instituts für germanische Philologie, feierlich begrüßt.

Mit seinem Plenarvortrag eröffnete Prof. Dr. habil. Waldemar Pfeiffer (Poznań) den inhaltlichen Teil der Konferenz. In seinem aufschlussreichen Beitrag akzentuierte er die Notwendigkeit, wichtige Erkenntnisse und Ergebnisse vieler, nicht nur sprachwissenschaftlicher, Bezugswissenschaften in die Glottodidaktik zu inkorporieren, wobei eine engere Verknüpfung zwischen universitärer Forschung und schulischer Praxis sowie eine verstärkte Berücksichtigung der pädagogischen und interkulturellen Kompetenz in der 
Fremdsprachenlehrerausbildung von besonders hoher Relevanz sind. Anschließend ergriff Dr. Sebastian Chudak (Poznań) das Wort, der sich in seinem Beitrag mit dem Stand der Diskussion um den Einsatz von neueren Medien im Fremdsprachenunterricht in ausgewählten polnischen Fachzeitschriften (darunter Glottodidactica, Neofilolog sowie Języki Obce w Szkole) kritisch auseinander setzte. Die nächste Referentin, Prof. Dr. habil. Camilla Badstübner-Kizik (Poznań), ging in ihrem Vortrag Inhaltsorientiertes Fremdsprachenlernen in medialen Umgebungen ausführlich auf das Konzept der "Erinnerungsorte“ ein, das mit Beispielen aus dem Kontext „DaF für polnische Lernende“ illustriert wurde.

Der zweite Teil der Plenarsitzung begann mit dem Vortrag von Dr. habil. Sylwia Adamczak-Krysztofowicz (Poznań) und Dr. habil. Antje Stork (Marburg), der von den ausgewählten Ergebnissen eines Kooperationsprojektes zwischen Studierenden aus Poznań und Marburg handelte. Dr. Magdalena Pieklarz (Olsztyn) präsentierte anschließend in ihrem Beitrag die Nutzungsmöglichkeiten gesprochener Lernerkorpora in der Fremdsprachendidaktik.

Aufgrund der hohen Anzahl der Konferenzteilnehmer wurde die Tagung in zwei Sektionen fortgesetzt, die von Dr. Ferran Suner (München) mit seinem Referat Kognitive Aspekte des Einsatzes von Hypertexten im Fremdsprachenunterricht sowie Dr. Matthias Springer (München) mit seinem Beitrag Geschichte und Landeskunde multimedial eröffnet wurden. Zum Schluss jener kurzen Referatsrunde sprach Dr. Marta Janachowska-Budych (Poznań) zum Thema Altes Medium in neuer Form. Literarische Texte und neue Medien im Fremdsprachenunterricht - Möglichkeiten und Vorteile des Einsatzes, während Mag. Monika Wilkowska (Poznań) über das mediengestützte integrierte Sprach- und Fachlernen (CLIL) referierte.

Am zweiten Konferenztag traten Dr. Elena Chudinova (Lipezk), Dr. Luiza Ciepielewska-Kaczmarek (Poznań), Mag. Magdalena Dudzińska (Poznań), Dr. Sebastian Dusza (Kraków), Mag. Konstancja Kuligowska (Poznań), Dr. Maciej Mackiewicz (Poznań), Prof. Dr. habil. Katalin Petneki (Szeged), Dr. Paweł Rybszleger (Poznań), Dr. Adam Sobek (Poznań) sowie Dr. Izabella Waibel (München) auf. In den Sektionen wurde u.a. über den Filmeinsatz im frühen DaF-Unterricht, über das mediengestützte handlungsorientierte Lernen, über die Förderung der interkulturellen Kompetenz durch den Medieneinsatz im Unterricht sowie über Lernplattformen und WWW-basierte soziale Netzwerke und deren Rolle im didaktischen Prozess diskutiert.

Die Plenarvorträge am zweiten Konferenztag begannen mit dem Beitrag von Mag. Martin Lange (Kiel), der die Technik des sog. „Virtual Classroom Managers“ präsentierte. In ihrem Beitrag reflektierte Dr. Izabela Marciniak (Poznań), über die Möglichkeiten, die Medienkompetenz bei angehenden Lehrern zu entwickeln.

Im Abschlusswort, das von Dr. Sebastian Chudak gesprochen wurde, wurde der Versuch unternommen, auf die während der Tagung diskutierten Themen sowie auf die sich daraus ergebenden Schlussfolgerungen noch einmal kurz einzugehen. Er machte noch einmal deutlich, dass Medien u.a. Raum für Reflexion über sprach- und kulturdidaktisch relevante Inhalte schaffen, die Begegnung mit einer Vielfalt von Texten verschiedener Art erleichtern, die auf eine viel komplexere Art und Weise miteinander verknüpft sind (Hypertexte) als das bei Lehrbuchtexten der Fall ist sowie zur Effizienzsteigerung des Lern- und Lehrprozesses (auch innerhalb der Lehrerausbildung) beitragen 
und ihr Mehrwert darin besteht, dass sie Lernende stark aktivieren und den Unterricht, an dem jene teilnehmen, intensivieren.

Während der Tagung wurden insgesamt 21 Referate gehalten, wobei nicht nur die polnischen Universitäten in Kraków, Olsztyn, Poznań und Toruń, sondern auch die deutschen Universitäten in Kiel, Marburg und München, die Öffentliche Verwaltungsakademie in Lipezk aus Russland, die Universität Szeged aus Ungarn vertreten waren.

Die Tagung hat die Möglichkeit zur Präsentation aufschlussreicher Forschungsergebnisse und zu lebhaften, fruchtbaren Diskussionen eröffnet. Da die Veröffentlichung der Beiträge vorgesehen ist, werden Interessierte jene nachlesen können. 